\title{
Arroo Oronat \\ ENFERMAGEM EM CIRURGIA AMBULATORIAL DE UM HOSPITAL ESCOLA: CLIENTELA, PROCEDIMENTOS E NECESSIDADES BIOLÓGICAS E PSICOSSOCIAIS ${ }^{1}$
}

\author{
Tatiane Vegette Pinto $^{2}$ \\ Izilda Esmênia MugliaAraújo ${ }^{3}$ \\ Maria Cecília Bueno Jayme Gallani ${ }^{4}$
}

Pinto TV, Araújo IEM, Gallani MCBJ. Enfermagem em cirurgia ambulatorial de um hospital escola: clientela, procedimentos e necessidades biológicas e psicossociais. Rev Latino-am Enfermagem 2005 março-abril; 13(2):208-15.

Este estudo descritivo teve como objetivos caracterizar o perfil dos pacientes atendidos no Centro Cirúrgico Ambulatorial de um Hospital Universitário do interior do Estado de São Paulo; identificar os procedimentos a que foram submetidos e identificar as necessidades biológicas e psicossociais desses pacientes. Estudo descritivo realizado por meio de entrevista semi-estruturada e consulta documental ao prontuário médico. Participaram do estudo 167 pacientes. Os resultados evidenciaram que o perfil dos pacientes assistidos caracterizou-se pelo equilíbrio entre os sexos masculino e feminino, idade média de 51 anos, e baixo nível socioeconômico. Os procedimentos cirúrgicos e anestésicos mais freqüentes foram os da oftalmologia e de anestesia local, com ou sem sedação. Foram identificadas necessidades biológicas: alterações na pressão arterial, alterações eletrocardiográficas, uso de medicamentos, alergia a medicamentos, jejum prolongado, náuseas, vômito e dor; e psicossociais: preocupação, medo, ansiedade, desconforto com a espera pelo procedimento, e dúvidas ou desconhecimento sobre cuidados perioperatórios. A partir dos resultados obtidos, estratégias devem ser planejadas para melhoria da assistência prestada.

DESCRITORES: procedimentos cirúrgicos ambulatóriais; cuidados de enfermagem; enfermagem; cirurgia

\section{NURSING CARE IN AMBULATORY SURGERY AT A TEACHING HOSPITAL: PATIENTS, PROCEDURES AND BIOLOGICAL AND PSYCHOSOCIAL NEEDS}

This descriptive study aimed to characterize the profile of 167 subjects who were treated at the Ambulatory Surgical Center of a University Hospital in São Paulo State and procedures realized at the Ambulatory Surgical Center, as well as to identify the biological and psychosocial needs of these patients. Data were obtained through a semistructured interview and patients' files and were subject to descriptive analysis. The group was characterized by an equal number of individuals from both genders; average age was 51 years and socioeconomic levels were poor. The most common surgical and anesthetic procedures were ophthalmologic procedures and use of local anesthesia with or without sedation. The biological needs were: altered arterial pressure, electrocardiographic alterations, use of medication, allergy to medication, prolonged fasting, nausea, vomits and pain. The psychosocial needs were: worry, fear, anxiety, discomfort caused by waiting for the realization of procedures and doubts or lack of information concerning perioperative care.

DESCRIPTORS: ambulatory surgical procedures; nursing care; nursing; surgery

\section{ENFERMERÍA EN CIRUGÍA AMBULATORIA EN HOSPITAL ESCUELA: CLIENTELA, PROCEDIMIENTOS Y NECESIDADES BIOLÓGICAS Y PSICOSOCIALES}

Este estudio descriptivo tuvo como objetivos: caracterizar el perfil de las 167 personas que se sometieron a cirugía en quirófano ambulatorio de un Hospital Universitario en el estado de São Paulo, Brasil, así como los procedimientos realizados en dicho quirófano e identificar las necesidades biológicas y psicosociales de estos pacientes. Las informaciones recopiladas por medio de entrevista semi-estructurada y consulta a los archivos fueron sometidas al análisis descriptivo. El grupo se constituyó de número igual de hombres y mujeres, con edad promedia de 51 años y bajo nivel socioeconómico. Los procedimientos quirúrgicos y anestésicos más frecuentes fueron los de oftalmología y de anestesia local con o sin sedación. Como necesidades biológicas fueron identificadas: alteraciones en la tensión arterial, alteraciones electrocardiográficas, uso de medicamentos y alergias a los mismos, ayuno prolongado, náusea, vómito y dolor; como necesidades psicosociales: preocupación, miedo, ansiedad, incomodo por la espera para realizarse el procedimiento y dudas o desconocimiento respecto a los cuidados perioperatorios.

DESCRIPTORES: procedimientos quirúrgicos ambulatorios; atención de enfermería, enfermería; cirugía

\footnotetext{
${ }^{1}$ Trabalho de Iniciação Científica financiado pelo SAE - Serviço de Apoio ao Estudante - Universidade Estadual de Campinas; ${ }^{2}$ Enfermeira; ${ }^{3}$ Enfermeira, Professor Assistente Doutor; ${ }^{4}$ Enfermeira, Professor Doutor, e-mail: ceciliag@fcm.unicamp.br. Faculdade de Ciências Médicas da Universidade Estadual de Campinas
} 


\section{INTRODUÇÃO}

Avanços na área farmacológica e das técnicas anestésicocirúrgicas acarretaram a popularização da cirurgia ambulatorial (CA) na década de $70^{(1-2)}$, com um crescimento expressivo do número de procedimentos ambulatoriais, quando comparados às cirurgias convencionais ${ }^{(2)}$, sendo qualificada por alguns autores como a "opção que deu certo"(3).

O Centro Cirúrgico Ambulatorial (CCA) é semelhante ao Centro Cirúrgico tradicional. A diferença consiste no fato de a alta ocorrer no mesmo dia do procedimento, o que implica uma série de vantagens como o pouco tempo de privação do convívio familiar, redução da ansiedade, menor risco de se contrair infecção hospitalar, diminuição do tempo de recuperação, além da economia para o hospital, instituições de seguro e governamentais ${ }^{(1,4)}$.

As cirurgias passíveis de serem realizadas no CCA são de pequena complexidade ${ }^{(3,5)}$, mediante técnicas anestésicas local, locoregional, de bloqueio (raqui, peridural) ou, ainda, a geral (inalatória el ou endovenosa).

Para avaliar a possibilidade de se submeter um paciente a CA, devem ser seguidos os critérios de seleção estabelecidos pelo Conselho Federal de Medicina, no artigo $1^{\circ}$, inciso II, da resolução n.1409/94, o qual determina que devam ser submetidos a CA os pacientes: a) com ausência de comprometimento sistêmico, seja por outras doenças ou pela cirurgia, e paciente com distúrbio sistêmico moderado, por doença geral compensada; b) que serão submetidos a procedimentos cirúrgicos que não precisem de cuidados especiais no pós-operatório; c) que disponham de acompanhante lúcido e previamente identificado ${ }^{(3)}$. Critérios como fatores fisiológicos, fatores sociais e psicológicos, avaliação clínica e laboratorial, também devem ser considerados.

O enfermeiro é indispensável no CCA, desde o início, no projeto de construção da unidade, visando garantir a distribuição estratégica de salas, unidades e equipamentos, de modo a resultar em fluxograma lógico e seguro, até a administração e prestação de assistência direta e indireta ao paciente, em três momentos distintos: pré, trans e pós-operatório.

A atuação do enfermeiro no cuidado direto ou indireto ao paciente em CA é peculiar; pois tem que ser ágil e dinâmica, contemplando a complexidade das diferentes demandas do paciente nos três períodos, direcionando o cuidado para que o paciente consiga atingir, o mais breve possível, a condição clínica, emocional e social adequada para sua alta.

A caracterização de pacientes submetidos a CA em um dado serviço é essencial para que o enfermeiro possa identificar as necessidades prioritárias dos sujeitos atendidos, quer pela freqüência ou por seu significado ou conseqüências. A identificação dessas prioridades permite o estabelecimento de um determinado grupo de intervenções que visam ao restabelecimento mais rápido e seguro dos pacientes e, portanto, seu retorno mais precoce ao domicílio ${ }^{(6)}$.

\section{OBJETIVOS}

Visando obter subsídios para a elaboração de um protocolo mínimo de atendimento no CCA, bem como intervenções educativas do enfermeiro, o presente trabalho teve como objetivos:

- caracterizar o perfil dos pacientes;

- levantar os procedimentos realizados aos pacientes no atendimento Universitário do interior do Estado de São Paulo;

- identificar as necessidades biológicas e psicossociais dos pacientes que possam interferir na realização da cirurgia ou na alta hospitalar.

\section{MATERIAL E MÉTODO}

Trata-se de um estudo descritivo, cuja população foi constituída por sujeitos que PROCURARAM o CCA, submetidos ou não ao procedimento cirúrgico. Os critérios de seleção dos entrevistados foram: ser maior de 18 anos ou estar autorizado pelo responsável, estar em condições de comunicar-se e concordar em participar da pesquisa, segundo termo de consentimento livre esclarecido. Aamostra, determinada por procedimento não probabilístico, por conveniência, foi constituída por 167 sujeitos, atendidos no período de dezembro de 1998 a fevereiro de $1999^{*}$.

Para a coleta dos dados, foi empregada a técnica de entrevista semi-estruturada e consulta ao prontuário do paciente. Para nortear a obtenção dos dados, foi utilizado o instrumento para avaliação perioperatória desenvolvido anteriormente* ${ }^{\star \star}$ O instrumento consta de três partes: identificação do paciente, avaliação pré-operatória, e

\footnotetext{
* Destaca-se que houve um recesso no período compreendido entre 23 de dezembro de 1998 a 4 de janeiro de 1999 , durante o qual o CCA interrompeu suas atividades

** Instrumento desenvolvido por Gallani, MCBJ (1995) e utilizado na Disciplina Enfermagem de Enfermagem em Centro Cirúrgico, do Departamento de Enfermagem da Faculdade de Ciências Médicas da UNICAMP
} 
recuperação pós-anestésica. Ressalta-se que as informações referentes ao quadro clínico e cuidados prestados (diagnóstico médico, cirurgia proposta, tipo de anestesia, valores mensurados de sinais vitais e de exames de sangue e intercorrências durante o procedimento cirúrgico) foram obtidos a partir da consulta ao prontuário. Os dados foram inicialmente transportados para o Programa Excel for Windows/ 95 e, posteriormente, submetidos à análise descritiva. O estudo foi aprovado pelo Comitê de Ética em Pesquisa da Faculdade de Ciências Médicas - UNICAMP (Parecer CEP n.166/98).

\section{RESULTADOS}

\section{Caracterização dos sujeitos}

Dos 167 pacientes entrevistados, 20 (12,0\%) tiveram suas cirurgias suspensas por falta de horário cirúrgico, falta de material, ou por condição clínica desfavorável (elevação da pressão arterial não revertida com repouso e sedação), aos quais não se aplicou a terceira parte do instrumento. Outros seis pacientes (3,6\%) encontravam-se internados previamente a CA; nesse caso, não foram aplicadas as questões de número 5 a 11 do item de avaliação pós-operatória. $A$ questão referente à auto-avaliação sobre a experiência nessa cirurgia não foi aplicada a seis crianças. A descrição sociodemográfica dos 167 sujeitos é apresentada na Tabela 1. As ocupações profissionais

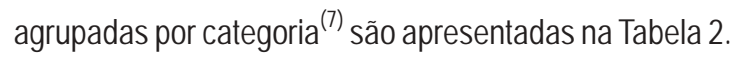

Tabela 1 - Distribuição das características sociodemográficas dos pacientes ( $n=167)$ entrevistados. Campinas. 1998-1999

\begin{tabular}{lcc}
\hline & $\mathrm{n}(\%)$ & média $\left(\mathrm{dp}^{*}\right)$ \\
Idade & & \\
\hline Sexo & & \\
$\quad$ Masculino & & \\
Feminino & $86(51,3)$ \\
Estado Civil & $81(48,5)$ & \\
Sem parceiro & $52(31,1)$ \\
Com parceiro & $115(68,9)$ \\
Religião & $120(72,0)$ \\
Católica & $18(10,8)$ \\
Evangélica & $29(17,2)$ \\
Outras & $34(20,4)$ \\
Escolaridade & $113(67,6)$ \\
Analfabeto & $11(6,6)$ \\
Ensino Fundamental & $9(5,4)$ \\
Ensino Médio &
\end{tabular}

* $\mathrm{dp}=$ desvio padrão
Tabela 2 - Distribuição dos pacientes $\left(n=161^{*}\right)$ de acordo com a categoria profissional $^{(6)}$. Campinas. 1998-1999

\begin{tabular}{|c|c|}
\hline Categoria & $\mathrm{n}(\%)$ \\
\hline CATEGORIA I & \\
\hline Profissionais liberais e assemelhados & $1(0,6)$ \\
\hline CATEGORIA II & \\
\hline $\begin{array}{l}\text { Posições mais baixas de supervisão ou inspeção de } \\
\text { ocupações não manuais, proprietários de pequenas } \\
\text { empresas comerciais / industriais / agropecuárias }\end{array}$ & $8(5,0)$ \\
\hline CATEGORIA III & \\
\hline $\begin{array}{l}\text { Ocupações não manuais de rotina e assem elhadas } \\
\text { CATEGORIA IV }\end{array}$ & $7(4,3)$ \\
\hline $\begin{array}{l}\text { Supervisão de trabalhos manuais e ocupações } \\
\text { assemelhadas }\end{array}$ & $2(1,2)$ \\
\hline CATEGORIA V & \\
\hline $\begin{array}{l}\text { Ocupações manuais especializadas e assem elhadas } \\
\text { CATEGORIA VI }\end{array}$ & $22(13,7)$ \\
\hline $\begin{array}{l}\text { Ocupações manuais não especializadas } \\
\text { CATEGORIA VII }\end{array}$ & $12(7,5)$ \\
\hline $\begin{array}{l}\text { Aposentados e pensionistas } \\
\text { CATEGORIA VIII }\end{array}$ & $65(40,4)$ \\
\hline Donas-de-casa & $31(19,3)$ \\
\hline CATEGORIA IX & \\
\hline Estudantes & $9(5,6)$ \\
\hline CATEGORIA $X$ & \\
\hline Desempregados & $4(2,4)$ \\
\hline
\end{tabular}

*foram excluídos dessa classificação seis pacientes pediátricos, abaixo da idade escolar

A distribuição dos pacientes submetidos a cirurgia ambulatorial segundo a especialidade cirúrgica, diagnósticos médicos mais freqüentes e procedimentos realizados é apresentada na Tabela 3.

Tabela 3 - Distribuição da freqüência relativa dos procedimentos cirúrgicos de acordo com os diagnósticos médicos e as especialidades médicas realizados no CCA. Campinas, 1998-1999

\begin{tabular}{|c|c|c|c|}
\hline $\begin{array}{l}\text { Especialidade } \\
\text { médica }\end{array}$ & $(\%)$ & $\begin{array}{l}\text { Diagnósticos mais } \\
\text { freqüentes }\end{array}$ & Procedimentos realizados \\
\hline $\begin{array}{l}\text { Oftalmologia } \\
\text { geral ou plástica }\end{array}$ & 58,7 & $\begin{array}{l}\text { catarata, deslocamento } \\
\text { de retina, pterígio, ptose } \\
\text { palpebral, dacriocistite, } \\
\text { glaucoma, obstrução do } \\
\text { canal lacrimal, } \\
\text { deslocamento de lente } \\
\text { intra-ocular, estrabismo, } \\
\text { tumores }\end{array}$ & $\begin{array}{l}\text { facectomia com implante de } \\
\text { lente intra-ocular, } \\
\text { dacriocistectomia, exerese de } \\
\text { pterígio, reconstrução de } \\
\text { canal lacrimal, retinopexia, } \\
\text { vitrectomia, trabulectomia, } \\
\text { exerese de tumores, implante } \\
\text { de peso de ouro em pálpebra }\end{array}$ \\
\hline Ortopedia & 9,0 & $\begin{array}{l}\text { lesões causadas por } \\
\text { acidente de trabalho e de } \\
\text { trânsito, artrose, rotura de } \\
\text { tendão, dedo em martelo } \\
\text { ou garra, hiperplasia } \\
\text { óssea, tumor de } \\
\text { metatarso, osteomielite, } \\
\text { alteração de menisco }\end{array}$ & $\begin{array}{l}\text { amputação, artroscopia, } \\
\text { artrostomia, exploração de } \\
\text { tendão, reconstrução de coto, } \\
\text { ressecção óssea, tenorrafia }\end{array}$ \\
\hline Dermatologia & 7,8 & 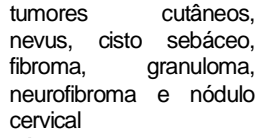 & $\begin{array}{l}\text { exereses de tumores } \\
\text { cutâneos, nevus, cisto } \\
\text { sebáceo, fibroma, granuloma, } \\
\text { neurofriboma e biópsia }\end{array}$ \\
\hline Gastrocirurgia & 7,2 & $\begin{array}{l}\text { hérnia, esofagite de } \\
\text { refluxo, hemorróida, } \\
\text { estenose de esôfago e } \\
\text { varizes esofágicas }\end{array}$ & $\begin{array}{l}\text { herniorrafia, endoscopia, } \\
\text { hemorroidectomia e esclerose } \\
\text { de varizes }\end{array}$ \\
\hline Proctologia & 2,4 & $\begin{array}{l}\text { abcesso, fissura ou fístula } \\
\text { anal }\end{array}$ & $\begin{array}{l}\text { drenagem de } \\
\text { esfincterectomia } \\
\text { fistulectomia }\end{array}$ \\
\hline Plástica & 6,6 & $\begin{array}{l}\text { hiperplasia mamária, } \\
\text { agenesia de pavilhão } \\
\text { auricular, quelóides e } \\
\text { cicatrizes, orelha de } \\
\text { abano, fratura nasal e } \\
\text { estenose oral }\end{array}$ & $\begin{array}{l}\text { adenomastectomia, otoplastia, } \\
\text { correção de orelha, enxertos, } \\
\text { reparo cutâneo e zetaplastia }\end{array}$ \\
\hline Urologia & 4,2 & $\begin{array}{lr}\text { esterilidade } & \text { ou } \\
\text { infertilidade, } & \text { fimose, } \\
\text { hiperplasia de frênulo, } \\
\text { impotência e insuficiência } \\
\text { renal crônica }\end{array}$ & $\begin{array}{l}\text { biópsia testicular, postectomia, } \\
\text { exerese de hiperplasia de } \\
\text { frênulo, implante de prótese } \\
\text { peniana e fístula artério- } \\
\text { venosa }\end{array}$ \\
\hline Vascular & 2,4 & varizes & $\begin{array}{l}\text { Exerese de colaterais e } \\
\text { safenectomia }\end{array}$ \\
\hline $\begin{array}{l}\text { Otorrino- } \\
\text { laringologia }\end{array}$ & 1,8 & $\begin{array}{l}\text { perfuração de tímpano, } \\
\text { pólipo nasal, pólipo } \\
\text { auricular }\end{array}$ & $\begin{array}{l}\text { reconstrução de tímpano, } \\
\text { exerese de pólipo nasal e } \\
\text { exerese de pólipo auricular }\end{array}$ \\
\hline
\end{tabular}


Dados da avaliação pré-operatória

Necessidades biológicas

No item do instrumento, voltado à avaliação pré-operatória, pôde ser constatado, em consulta ao prontuário do paciente, que todos possuíam o diagnóstico médico e proposta cirúrgica, e apenas 34,13\% (57/167) possuíam avaliação mais completa, englobando o estado geral, investigação sobre doenças (doenças cardiovasculares, diabetes, distúrbio pulmonar, hemofilia e febre reumática) e antecedentes cirúrgicos. A abordagem sobre algum tipo de alergia ocorreu apenas no momento da admissão do paciente para a cirurgia.

As avaliações gerais realizadas junto aos pacientes no préoperatório encontram-se descritas na Tabela 4. Destaca-se que, dos 85 sujeitos submetidos ao exame de eletrocardiograma, 15,3\% (13/ 85) apresentavam algum tipo de alteração. Todos os resultados dos exames laboratoriais realizados encontravam-se dentro dos parâmetros de normalidade.

Tabela 4 - Distribuição das freqüências absoluta (n) e relativa (\%) das avaliações pré-operatórias realizadas (n=167). Campinas. 1998-1999

\begin{tabular}{lc}
\hline \multicolumn{1}{c}{ TIPO DE AVALIAÇÃO } & $\mathrm{n}(\%)$ \\
\hline Eletrocardiograma & $85(50,9)$ \\
Dosagem de glicemia & $89(53,3)$ \\
Dosagem de hemoglobina & $103(61,7)$ \\
Dosagem de hematócrito & $102(61,0)$ \\
Mensuração da pressão arterial & $134(80,2)$ \\
Mensuração da temperatura & $06(4,1)$ \\
Mensuração da freqüência respiratória & - \\
Mensuração da freqüência cardíaca & - \\
\hline
\end{tabular}

Todos os pacientes entrevistados apresentavam higiene pessoal satisfatória. Sete pacientes $(4,2 \%)$ deveriam ter realizado a tricotomia, e todos a haviam executado de maneira adequada. Dos 114 pacientes que usavam prótese dentária, apenas 9,6\% (11/114) não haviam retirado, alegando falta da informação ou esquecimento. Não houve suspensão de cirurgia por inadequação do preparo préoperatório.

O tempo de jejum encontrado variou de 2 a 19 horas, com valor médio de 8 horas para os 167 pacientes. Dos pacientes entrevistados, 50,90\% (85/167) utilizavam regularmente algum tipo de medicação (anti-hipertensivos, hipoglicemiantes, antiagregantes plaquetários, tranqüilizantes dentre outros) e 11,40\% (19/167) relatavam algum tipo de reação alérgica a medicamentos.

Necessidades psicossociais

A Tabela 5 apresenta uma síntese da freqüência dos aspectos psicossociais identificados no estudo.
Tabela 5 - Distribuição dos aspectos psicossociais verificados nos pacientes do CCA no período pré-operatório. Campinas, 1998-1999

\begin{tabular}{lc}
\hline \multicolumn{1}{c}{ ASPECTOS PSICOSSOCIAIS } & $\mathrm{n}^{*} / \%$ \\
\hline -Experiência cirúrgica prévia & $135 / 167(80.8)$ \\
- Cirurgia ambulatorial & $19 / 135(14,1)$ \\
-Conhecimento sobre diagnóstico médico & $155 / 167(92,8)$ \\
-Conhecimento sobre cirurgia proposta & $161 / 167(96,4)$ \\
-Conhecimento sobre tipo anestesia & $163 / 167(97,6)$ \\
-Preocupação & $79 / 161(49,9)$ \\
- com o medo da dor/anestesia/cirurgia & $37 / 79(47,0)$ \\
- com a família & $18 / 79(22,8)$ \\
- com espera do procedimento & $12 / 79(15,0)$ \\
\hline${ }^{*}$ = número de casos observados/número de sujeitos em que a variável foi \\
pesquisada
\end{tabular}

Dados da avaliação pós-operatória

Necessidades biológicas

A terceira parte do instrumento contempla os dados referentes à recuperação pós-anestésica, a readaptação ao ambiente e preparo para alta hospitalar do paciente submetido a cirurgia ambulatorial. Em relação às intercorrências na sala de operações, houve relato de complicações em apenas 2,0\% (3/147), que foram: hemorragia peribulbar e a rotura de câmara posterior durante a aspiração do cristalino, em cirurgias oftalmológicas.

Observou-se que 55,8\% (82/147) dos entrevistados foram submetidos a anestesia local e sedação; 29,9\% (44/147) a anestesia local; 10,2\% (15/147) a geral. Bloqueio peridural, raquimedular e braquial juntos corresponderam a 2,0\% (3/147) das anestesias previstas. Os casos nos quais foram empregadas a anestesia geral associada a bloqueio (1,4\% ou 2/147) e a anestesia local associada a geral $(0,7 \%$ ou $1 / 147)$ tinham como a proposta inicial a realização de anestesia local e o bloqueio, respectivamente, porém complicações no procedimento cirúrgico levaram à modificação do tipo de anestesia. As substâncias utilizadas para a anestesia local com sedação foram a lidocaína (anestésico local), e o diazepam (sedativo) ingerido assim que o paciente era chamado para a sala de preparo pré-operatório.

Os pacientes que receberam anestesia local ou local com sedação não permaneceram na sala de recuperação pós-anestésica (SRPA), sendo liberados após orientação sobre os cuidados no pósoperatório, retorno e prescrição médica. Somente os pacientes submetidos a bloqueio e anestesia geral foram admitidos na sala de recuperação anestésica.

Apenas 20 prontuários continham anotações de enfermagem referentes aos sinais vitais na admissão na sala de recuperação pósanestésica, readaptação do paciente ao ambiente e sua condição para alta. É válido lembrar que, dos 147 pacientes que foram submetidos a procedimento cirúrgico, 55,8\% (82/147) receberam anestesia local associada a sedação, recebendo alta, portanto, sem permanecer na SRPA. Os pacientes que permaneceram na SRPA foram aqueles que apresentaram complicações no pós-operatório ou foram submetidos a procedimentos que requeriam avaliação e cuidados específicos. A 
avaliação e os cuidados de enfermagem realizados junto a esses pacientes, na maioria das vezes, não foram registrados nos respectivos prontuários.

Em relação às condições apresentadas pelos pacientes para alta, observou-se que nenhum deles apresentou sangramento/ drenagem maior que o esperado, ou dor não aliviada com uso de medicamentos; $4,1 \%$ apresentaram cefaléia e foram medicados com analgésico intra-muscular; assim como os 2,0\% que referiram dor maior que 0 esperado, relacionada à cirurgia; $4,1 \%$ apresentaram tontura e $0,7 \%$ referiram náusea ou vômito.

\section{Necessidades psicossociais}

Quando questionados sobre os cuidados a serem tomados em casa, $86,5 \%$ dos entrevistados foram capazes de relacioná-los; dos $13,5 \%$ que não se encontravam esclarecidos, mais da metade relacionou os cuidados que foram fornecidos anteriormente, e alguns não haviam sido orientados até o momento da entrevista.

Os 135 pacientes adultos que retornariam para casa, referiram estar preparados para o retorno. Dentre as justificativas apontadas, constatou-se que 70,9\% (96/135) avaliavam seu estado como suficientemente bom para o retorno; 19,3\% (26/135) atrelaram sua boa condição à ausência de dor (algumas vezes garantida pelo uso de analgésicos); 4,4\% (6/135) associavam sua boa condição à "simplicidade" da cirurgia; 3,0\% (4/135) destacaram o conhecimento dos cuidados a serem tomados, como o motivo para a segurança na alta e 2,2\% (3/134) julgavam que se recuperariam melhor em casa.

Quando indagados se gostariam de esclarecer alguma dúvida 84,4\% (114/135) referiram não apresentar dúvidas. Quando a resposta foi afirmativa, foram constatadas dúvidas diversas, relacionadas ao problema de saúde, procedimento cirúrgico e aos cuidados no pósoperatório como por exemplo: Retina é doença?, Infertilidade resolve com remédio?, Como é um transplante de córnea?, A cirurgia teve êxito?, O organismo absorve os pontos?, Em quanto tempo voltarei a trabalhar?, Qual é a alimentação e como é o repouso adequado para os próximos dias?, Onde posso dormir para poder fazer o retorno amanhã?

Dos 141 que retornariam para o lar, o cônjuge ou o namorado foram referidos como acompanhantes por 39,0\% (55/141) dos entrevistados; outros 28,4\% (40/141) estavam acompanhados de seus filhos; $12,0 \%$ (17/141), de seus pais, e 10,6\% (15/141), dos irmãos. Amigos foram acompanhantes de 4,3\% (6/141) dos pacientes e 5,7\% (8/141) estavam acompanhados de outros parentes (tios, sobrinhos, avôs e primos). Dos 141 pacientes que se encontravam com acompanhantes no pós-operatório, 78,0\% (110/141) continuariam com esses mesmos acompanhantes como cuidadores no domicílio após a alta. Dos demais, 31 sujeitos, somente dois não tinham pessoas para assumir o papel de cuidador em seus domicílios.
O presente estudo apontou que, dos 135 pacientes aos quais foi solicitada avaliação da experiência cirúrgica, 81,5\% (110/135) julgaram-na boa, e outros 11,1\% (15/135), excelente. A experiência positiva foi atribuída à boa acolhida, ao fato de "sentir-se bem", à evolução dentro do previsto, à confiança na equipe médica e de enfermagem. Alguns pacientes, mesmo ressaltando aspectos que seriam negativos, como a demora para ser chamado à sala de operações depois de preparado; medo em relação ao procedimento cirúrgico ou à técnica anestésica, consideraram positiva a experiência. $\mathrm{A}$ manifestação de ter sido uma experiência regular foi de 7,4\% (10/ 135); e estes justificaram pela insegurança em relação ao êxito da cirurgia, pela dor, pela tontura ou pelo sentimento de pesar pela amputação sofrida.

As opiniões emitidas pelos pacientes sobre a permanência em casa na véspera da cirurgia e sobre o retorno ao lar, mostraram uma avaliação positiva em 96,5\% (130/135) e 95,7\% (129/135), respectivamente. As justificativas mais freqüentes para os dois itens foram: sentir-se melhor em casa; estar com a família; tranqüilidade pessoal/familiar porque a cirurgia é simples; pelo receio da internação; porque liberam leitos para outros casos e pelo menor risco de infecção. Um número reduzido de pacientes mostrou-se desfavorável a permanecer em casa antes do procedimento $(3,5 \%$ ou $5 / 135)$ ou a retornar para o lar logo após a cirurgia $(4,3 \%$, ou $6 / 135)$, alegando problemas pela distância e, portanto, para o retorno, ou por julgarem mais adequados os cuidados prestados durante a internação.

Sobre os cuidados no domicílio após a alta, 86,6\% (117) 135) conseguiram relacioná-los. Os cuidados mais referidos, nos casos de cirurgia oftalmológica, foram sobre a troca de curativos, instilação de colírio, não realização de movimentos bruscos da cabeça, não carregar peso, cuidado ao tossir, não coçar a região e não dormir sobre o lado operado.

Cuidados com curativo, tala, repouso, posicionamento do membro e fisioterapia foram os cuidados citados pelos pacientes de cirurgia ortopédica. Nas cirurgias vasculares, os cuidados mencionados foram referentes ao uso de meia elástica, repouso e posicionamento do membro. Já nas cirurgias de otorrinolaringologia, os cuidados mais mencionados foram quanto ao uso de medicação, curativo e higiene local.

Entre os cuidados mais citados pelos pacientes submetidos a procedimentos de cirurgia plástica e dermatológica podem-se salientar os cuidados de higiene com a lesão, cuidados para não fazer esforço e evitar a exposição ao sol da região operada. Nos casos de gastrocirurgia, os cuidados referidos foram sobre alimentação, esforço físico e necessidade de repouso. Nas cirurgias urológicas, os cuidados foram referentes à necessidade de repouso, de abstinência sexual, higiene local e utilização de suspensório escrotal. 


\section{DISCUSSÃO}

Os resultados deste estudo mostraram que, na população estudada, houve o predomínio de pacientes de idade adulta, com parceiro, de condição socioeconômica menos favorecida, como evidenciado pela alta prevalência do pouco tempo de estudo, aposentados/pensionistas ou realização de atividades laborais não especializadas. Em relação ao nível de escolaridade e situação socioeconômica, o grupo estudado reflete a maioria de pacientes atendidos em hospital universitário que presta assistência por meio do Sistema Único de Saúde: predominantemente formada por sujeitos com nível socioeconômico inferior e baixa escolaridade. Apenas 28,1\% dos pacientes procediam da cidade de Campinas, sendo os demais procedentes até de outros estados, embora tenha sido evidenciado que menos de 4,3\% tenham se queixado da dificuldade de retorno para o lar, relacionada à distância. A demanda por serviços públicos de referência, como um hospital escola, leva os pacientes a procurarem meios para superar a questão da distância que seria um critério de exclusão para CA, conseguindo permanência provisória em casa de amigos ou familiares, durante o período de tratamento.

Embora tenha sido constatado o predomínio de cirurgias oftalmológicas e de anestesia local, com ou sem associação de sedação, com emprego de xylocaína e diazepam, houve uma diversidade de procedimentos realizados, que coincide com a literatura ${ }^{(3)}$ e aponta para a necessidade de determinação de protocolos mínimos de cuidados para cada uma das especialidades.

A avaliação pré-operatória do paciente em relação aos sinais vitais e exames laboratoriais é indispensável, pois permite a detecção de afecções até então não suspeitadas, correção das alterações de modo a possibilitar o tratamento cirúrgico ou até contra-indicar 0 procedimento, além de contribuir, de modo significativo, para a redução da ansiedade pré-operatória, da dor pós-operatória, e, principalmente, da morbidade peri-operatória ${ }^{(8-9)}$. 0 baixo índice de registros pela equipe de saúde, tanto em relação à avaliação clínica e dos cuidados prestados, como à verificação de sinais vitais, deixa evidente a necessidade de serem empreendidos esforços para a determinação de padrões mínimos de conduta e de registro da avaliação, bem como dos cuidados prestados ao paciente nesses períodos. Seria adequado que 0 desenvolvimento desses padrões se realizasse a partir das recomendações da literatura e das observações da práxis e que sua implementação fosse realizada sob a forma de educação continuada, buscando obter 0 envolvimento e o compromisso de todos os profissionais.

A adoção do jejum pré-operatório prolongado em pacientes eletivos tem sido contra-indicada na literatura, sendo alegado não haver base científica que o justifique, uma vez que a ingestão de pequena quantidade de líquidos, até três horas antes da cirurgia, não altera 0 risco de regurgitação inesperada e vômito, recomendando a ingestão livre de água até duas horas antes da indução anestésica ou $150 \mathrm{ml}$ de café/chá/suco de fruta até três horas antes da cirurgia ${ }^{(10)}$. 0 jejum prolongado resulta em desconforto e irritabilidade (principalmente em crianças), depleção de líquidos e suas conseqüências: desidratação, hipotensão grave durante a indução anestésica por hipovolemia relativa, aumento do risco de vômitos no pós-operatório e de ocorrência de hipoglicemia no peri-operatório ${ }^{(10)}$. Considerando-se que, neste estudo, foi observado um período médio de oito horas de jejum, com períodos de até 19 horas, recomenda-se que essa questão seja revista na unidade.

Na literatura, há a recomendação de que o enfoque da assistência de enfermagem no CCA seja no bem-estar do paciente, em sua segurança, no seu conforto, na educação e na continuidade do cuidado, apontando o conhecimento do paciente sobre os cuidados necessários, como método para avaliar a efetividade da atividade educativa $^{(11-12)}$. Neste estudo, constatou-se que maioria dos entrevistados conhecia, ou tinha algumas informações sobre 0 diagnóstico médico, cirurgia proposta, tipo de anestesia a que seria submetido; havia realizado os cuidados mínimos pré-operatórios, bem como conseguia listar a maior parte dos cuidados a serem tomados após a cirurgia. Esses achados se contrapõem ao resultado de estudo anterior $^{(11)}$ realizado com pacientes em CA, onde foi observado que nenhum dos treze pacientes entrevistados possuía informações sobre anestesia, cirurgia e cuidados no pós-operatório, o que pareceu ser uma fonte de estresse para o grupo estudado.

Os resultados aqui observados levam a inferir a existência de uma atividade educacional mínima para esses sujeitos nos períodos pré e/ou pós-operatórios. É importante destacar, entretanto, que dentre as especialidades, os pacientes que se mostravam mais esclarecidos em relação aos cuidados foram os da oftalmologia, que realiza um trabalho específico de educação em saúde, desenvolvido por um grupo de enfermeiros e que prepara os pacientes para a cirurgia ${ }^{(13)}$. De acordo com a literatura ${ }^{(12)}$, a avaliação dos conhecimentos do paciente sobre 0 cuidado é um crítico indicador da qualidade do trabalho educativo realizado. Portanto, devem ser estudadas maneiras de estender o trabalho educativo a todos os pacientes submetidos a CA, seja pela enfermeira do CA ou do ambulatório de onde provém 0 paciente.

Embora seja descrito na literatura que o paciente submetido a CA apresenta uma diminuição da ansiedade, porque avalia esse tipo de procedimento como menos complexo que as cirurgias convencionais $^{(14)}$, constatou-se, neste estudo, que metade do grupo estudado ainda apresentava preocupações no período pré-operatório. Esses achados são corroborados pelos resultados de estudo recente realizado juntamente a pacientes submetidos a cirurgia ambulatorial $\mathrm{e}$ reforçam a necessidade de avaliação do comportamento físico e 
psicossocial geral, com destaque para alterações sensoriais, condição emocional, interação com a família e cooperação com instruções sobre preparo pré-operatório recebidas anteriormente; para implementação de intervenções que visem reduzir o estresse neste período ${ }^{(12,15)}$.

A primeira fase do pós-operatório consiste na recuperação do paciente após a anestesia, devendo, portanto, serem avaliados: freqüência respiratória, freqüência cardíaca, pressão arterial, nível de consciência, coloração da pele e grau de atividades espontâneas, principalmente quando empregada a anestesia geral. Na segunda fase do pós-operatório, ocorre a readaptação do paciente ao ambiente, identificada por atitudes como: sentar, levantar, deambular ${ }^{(12)}$. Novamente, houve pequena porcentagem de casos em que foi realizada e registrada tal avaliação, apesar de sua importância legal e de servir para a equipe como guia para prestação de cuidados, apontando para a necessidade de revisão dessa prática.

Outra fase do pós-operatório, corresponde à avaliação do paciente quanto aos critérios para alta: orientação auto e alopsíquica; estabilidade dos sinais vitais por pelo menos 60 minutos; ausência de náusea e vômitos; capacidade de ingerir líquidos; capacidade de locomoção como antes, se a cirurgia permitir; sangramento mínimo ou ausente; ausência de dor de grande intensidade; ausência de sinais de retenção urinária; que 0 paciente e acompanhante tenham conhecimento verbal e por escrito dos cuidados relativos ao pósoperatório e como proceder diante de complicações ${ }^{(3,12,16-17)}$. Neste estudo, foi observada baixa incidência de condições que contraindicassem a alta, o que não descarta, porém, a necessidade de 0 enfermeiro identificar as situações de risco e de tomar as medidas profiláticas ou de controle o mais precoce possível.

Na cirurgia ambulatorial, éimportante que se faça a educação do paciente e de seu acompanhante, preparando-os para assumirem os cuidados pré e pós-operatórios, sendo importante que a mesma pessoa que receba as orientações antes da cirurgia, permaneça com o paciente no dia, e preste os cuidados no domicílio. Neste estudo, todos os pacientes relataram estar preparados para o retorno ao lar, referindo poucas dúvidas e demonstrando conhecimento sobre os cuidados mínimos a serem executados no domicílio, o que permite avaliar as orientações sobre o pós-operatório como adequadas, devendo ser incentivadas.

A avaliação que o paciente faz de sua experiência cirúrgica é um indicador da qualidade do cuidado prestado e das áreas que precisam ser melhoradas ${ }^{(12,18)}$. A maioria dos pacientes estudados (92,8\%) julgou a experiência como positiva (boa ou excelente). Dessa maneira, os aspectos que contribuíram para a boa avaliação da experiência devem ser reforçados junto à equipe de saúde, para a manutenção e otimização da conduta, levando-se em consideração os aspectos avaliados como negativos (espera, dor, sofrimento, medo, incerteza), para as quais devem ser elaboradas e implementadas intervenções, principalmente educativas ${ }^{(19)}$, que permitam minimizálos.

\section{CONCLUSÕES}

Os dados obtidos neste estudo permitiram evidenciar que os pacientes atendidos no CCA em estudo, caracterizaram-se pelo equilíbrio entre o sexo masculino e feminino, idade média de 51 anos, estado civil casado, com baixo nível socioeconômico. Os procedimentos cirúrgicos mais freqüentes foram os de oftalmologia. Houve, ainda, uma discreta concentração de procedimentos nas especialidades: ortopedia, dermatologia e gastrocirurgia, com pequena prevalência de procedimentos da cirurgia plástica, urologia, cirurgia vascular e otorrinolaringologia. Em relação aos procedimentos anestésicos, o mais prevalente foi anestesia local com sedação. As necessidades biológicas evidenciadas pelos pacientes do estudo foram: alterações na pressão arterial, alterações eletrocardiográficas, uso de medicamentos para tratamento de co-morbidades, história de alergia a medicamentos, jejum prolongado (pré-operatório), náuseas, vômito e dor (pós-operatório). As necessidades psicossociais identificadas foram preocupação com a família, medo (da dor, da cirurgia e da anestesia), ansiedade e desconforto com a espera pelo procedimento, e relato de dúvidas ou desconhecimento sobre cuidados perioperatórios. Destaca-se a ausência de documentação sistemática das condições clínicas dos pacientes nesse período, bem como das condutas implementadas e sua avaliação.

Frente aos resultados observados, recomenda-se 0 estabelecimento de padrões mínimos de cuidado para cada especialidade, procurando atender às necessidades biológicas e psicossociais evidenciadas neste estudo, por meio da educação continuada, com incentivo à realização de registros de intervenções e condições peri-operatórias do paciente, pela equipe de saúde.

\section{REFERÊNCIAS BIBLIOGRÁFICAS}

1. Smeltzer SC, Bare BG. Brunner \& Suddarth - tratado de enfermagem médico-cirúrgica. $8^{\mathrm{a}}$ ed. Rio de Janeiro (RJ): Guanabara Koogan; 1994.

2. Brockway PM. The ambulatory surgical nurse. Nurs Clin North Am 1997; 32:387-94.

3. Silva MDAA, Rodrigues AL, Cesaretti IUR. Cirurgia ambulatorial. In: Silva MDAA, Rodrigues AL, Cesaretti IUR. Enfermagem na unidade de centro cirúrgico. $2^{\text {a }}$ ed. São Paulo (SP): EPU; 1997. p.161-6. 4. Peres MAO. Implementação da cirurgia ambulatorial e simplificada em hospital comunitário. [tese]. Campinas (SP): Faculdade de Ciências Médicas/UNICAMP; 1992. 
5. Praça MT, Azevedo FL. Relato de experiência sobre atuação da enfermeira na unidade de centro cirúrgico ambulatorial. Anais do $2^{\circ}$ Congresso Brasileiro de Enfermagem em Centro Cirúrgico Ambulatorial; 1995 Julho 4-7; São Paulo, Brasil, 1995.

6. Galdeano LE, Rossi LA. Construção e validação de instrumentos de coleta de dados para período perioperatório de cirurgia cardíaca. Rev Latino-am Enfermagem 2002; 10(6):800-4.

7. Unicamp (BR). Manual do candidato: vestibular nacional da UNICAMP. Campinas (SP); UNICAMP; 1996.

8. Aun F, Bevilacqua RG. Manual de Cirurgia. São Paulo (SP): EPU; 1995.

9. Gusman PB, Nascimento P Junior, Castiglia YMM, Amorim RB. Avaliação pré-anestésica ambulatorial. Rev Bras Anestesiol 1997; 47(6):522-7.

10. Macuco MV. Jejum pré-operatório: validade de critérios. Rev Bras Anestesiol 1998; 48(4):295-308.

11. Guido LA. Acompanhamento ao cliente cirúrgico ambulatorial fundamentado em King: relato de experiência em aplicar um marco conceitual e propor uma assistência de enfermagem alicerçada na perspectiva de humanização. Rev SOBECC 1996; 1(1):8-13.

12. Watson DS, Sangermano CA. Cirurgia ambulatorial. In: Meeker $\mathrm{MH}$, Rothrock JC. Cuidados de enfermagem ao paciente cirúrgico. 10ª ed. Rio de Janeiro (RJ): Guanabara Koogan; 1997.

13. Cárnio AM, Cintra FA, Tonussi JAG. Orientação pré-operatória a pacientes com catarata e indicação de cirurgia ambulatorial - relato de experiência. Rev Bras Enfermagem 1995; 48(1):39-45.

14. Kanamura AH. Unidade ambulatorial de pequena cirurgia - proposta de viabilização. Rev Paul Hosp 1981; 29(12):368-74.

15. Flório MCS, Galvão CM. Cirurgia ambulatorial: identificação dos diagnósticos de enfermagem no período perioperatório. Rev Latinoam Enfermagem 2003; 11(5):630-7.

16. Schmidt A, Bagatini A. Náusea e vômito pós-operatório: fisiopatologia, profilaxia e tratamento. Rev Bras Anestesiol 1997; 47(4):326-34.

17. Carvalho WA, Vianna PTG, Braz JRC. Náuseas e vômitos em anestesia: fisiopatologia e tratamento. Rev Bras Anestesiol 1999; 49(1):65-79.

18. Forbes ML, Brown HN. Developing an instrument for measuring patient satisfaction. AORN J 1995; 61(4):737-43.

19. Paula AAD, Carvalho EC. Ensino sobre pré-operatório a pacientes: estudo comparativo de recursos audiovisual (vídeo) e oral. Rev Latinoam Enfermagem 1997 julho/setembro; 5(3):35-42. 